\title{
Over-Expression of TBL1XR1 Indicates Poor Prognosis of Serous Epithelial Ovarian Cancer
}

\author{
Ming $\mathrm{Ma}^{1}$ and Nina $\mathrm{Yu}^{2}$ \\ ${ }^{1}$ Department of Oncology, Linyi People's Hospital, Linyi, Shandong, China \\ ${ }^{2}$ Department of Gynecology and Obstetrics, Linyi People's Hospital, Linyi, Shandong, China
}

Transducin $(\beta)$-like $1 \mathrm{X}$-linked receptor 1 (TBL1XR1) is a core component of the NCoR/SMRT transcription co-repressor complex, and its role in regulating cancer progression has been reported. Serous epithelial ovarian cancer (EOC) is the most common histological type of EOC. Here we explored the significance of TBL1XR1 expression in predicting outcomes of patients with serous EOC. Real-time quantitative PCR analysis showed that the expression level of TBL1XR1 mRNA was significantly higher in EOC tissues compared with adjacent non-tumorous tissues. The protein levels of TBL1XR1 in EOC tissues were assessed by immunohistochemistry, and the patients were classified into low-expression group $(n=62)$ and high-expression group $(n=54)$ according to the immunoreactivity. Prognostic significance of TBL1XR1 was evaluated by univariate and multivariate analyses, showing that over-expression of TBL1XR1 was correlated with poor prognosis. In addition, TBL1XR1 was positively associated with the lymph node metastasis of EOC. Because vascular endothelial growth factor (VEGF)-C is known as a critical mediator of lymph node metastasis, we measured the expression level of VEGF-C mRNA in EOC tissues and thus identified a positive correlation between TBL1XR1 and VEGF-C mRNA levels. Subsequently, using human EOC cell lines, we showed that silencing of TBL1XR1 decreased VEGF-C expression, suggesting that TBL1XR1 may function as an upstream regulator of VEGF-C in EOC. Furthermore, the proliferation and invasion of EOC cells were inhibited by TBL1XR1 silencing. In conclusion, TBL1XR1 overexpression may be an unfavorable prognostic factor for EOC. We also suggest that the TBL1XR1-VEGF-C axis may determine the EOC progression.

Keywords: epithelial ovarian cancer; lymph node; prognosis; TBL1XR1; VEGF-C

Tohoku J. Exp. Med., 2017 March, 241 (3), 239-247. (C) 2017 Tohoku University Medical Press

\section{Introduction}

Epithelial ovarian cancer (EOC) is the most lethal gynecological cancer and the second leading cause of cancer death among women worldwide (Siegel et al. 2014, 2015), and serous ovarian carcinoma is the most common histological type. EOC has often disseminated to the peritoneal cavity and been identified as advanced stages at the time of diagnosis, due to non-specific symptoms and lack of effective screening methods (Goff et al. 2000; Siegel et al. 2012). Despite current advances in surgery and adjuvant chemotherapy, the clinical outcome of patients with EOC remains unsatisfied. The 5-year overall survival rate was reported to be less than 50\% (Hunn and Rodriguez 2012), and below $30 \%$ for patients with advanced stages (Colombo et al. 2014). However, despite many clinical and basic research efforts, it is still a great challenge to identify novel biomarkers for early diagnosis, prediction or treatment of EOC.
The balance between ubiquitin ligases and deubiquitinases has attracted much attention in the study of malignancies. For example, ubiquitin-specific protease 33 (USP33) was shown to be down-regulated in breast cancer (YuasaKawada et al. 2009), lung cancer (Wen et al. 2014) and colorectal cancer (Liu et al. 2016b). On the other hand, we previously reported the enhanced expression of ubiquitinspecific protease 7 (USP7) in ovarian cancer (Ma and Yu 2016), which revealed that different ubiquitin-regulated enzymes may show completely different functions in tumor regulation. Here we investigated the expression and clinical significance of another ubiquitin-related protein, transducin $(\beta)$-like $1 \mathrm{X}$-linked receptor 1 (TBL1XR1), in EOC patients.

As an essential component of the SMRT (silencing mediator of retinoic acid and thyroid hormone receptors)/ $\mathrm{N}-\mathrm{CoR}$ (nuclear receptor corepressor) nuclear receptor corepressor complex, TBL1XR1 plays a crucial role in regulating the activation of corepressors by modulating the

Received November 22, 2016; revised and accepted March 6, 2017. Published online March 24, 2017; doi: 10.1620/tjem.241.239.

Correspondence: Nina Yu, Department of Gynecology and Obstetrics, Linyi People's Hospital, 27 Jiefang Road, Linyi, Shangdong 276000, China.

e-mail: ninayu2015@163.com 
ubiquitin status of SMRT/N-CoR complex (Zhang et al. 2002; Tomita et al. 2003, 2004; Yoon et al. 2003; Li and Wang 2008; Perissi et al. 2008). In other words, TBL1XR1 can promote the transcription processes mediated by transcription factors, including NF- $\kappa \mathrm{B}$, nuclear receptors (NRs), Wnt $/ \beta$-catenin and Notch pathways (Hoberg et al. 2004; Choi et al. 2011). Moreover, the functions of TBL1XR1 were distinct in different tumors. In prostate cancer, TBL1XR1 was reported to function as a tumor suppressor and inhibit tumor growth, perhaps through regulating androgen receptor (AR) signaling pathway (Daniels et al. 2014, 2016). However, TBL1XR1 has been reported to be highly expressed in cervical cancer (Wang et al. 2014), breast cancer (Li et al. 2014), nasopharyngeal carcinoma (Chen et al. 2014), hepatocellular carcinoma (Kuang et al. 2016), digestive cancers (Liu et al. 2016a; Zhou et al. 2016; Liu et al. 2017), and ovarian cancer (Wu et al. 2016). However, the significance of TBL1XR1 in predicting clinical outcomes of EOC patients has not been reported.

In the current study, we evaluated the expression of TBL1XR1 in serous EOC tissues and adjacent non-tumorous tissues, thereby showing that TBL1XR1 was highly expressed in serous EOC. In addition, the expression level of TBL1XR1 was correlated with lymph node metastasis and advanced FIGO stages. Subsequently, we verified the significant role of TBL1XR1 in predicting prognosis of serous EOC patients by univariate and multivariate analyses. Furthermore, our clinical studies and cell culture experiments demonstrated that TBL1XR1 can positively regulate the expression of vascular endothelial growth factor (VEGF)-C, which participates in the process of lymph node metastasis. Lastly, TBL1XR1 silencing dramatically down-regulated EOC cell proliferation and invasion, whereas TBL1XR1 transfection enhanced cell oncogenicity.

\section{Materials and Methods}

\section{Patients and samples}

Total 116 patients who were diagnosed with serous EOC and underwent R0 resection of primary tumors at Linyi People's Hospital were selected between June 2000 and June 2013. None of the patients received any chemoradiation or hormonal therapy before surgical resection. Patients' information including age, FIGO stage, pathological differentiation, lymph node status and pre-operative CA-125 level was retrieved. The paraffin-embedded specimens were obtained from Department of Pathology. Other 8 pairs of fresh-frozen serous EOC tissues and corresponding adjacent non-tumorous tissues were collected between 2014-2015, and were stored in liquid-nitrogen until use. This study was approved by the Ethics Committee of Linyi People's Hospital. Signed informed consent forms were obtained from all the patients.

\section{Immunohistochemistry (IHC) and IHC evaluation}

The IHC and IHC evaluation were performed as we described before (Ma and Yu 2016). Briefly, paraffin-embedded specimens were cut into $4-\mu \mathrm{m}$ sections, followed by deparaffinization with graded alcohol and antigen retrieval step. After blocked with non- immune goat serum, the slides were incubated with TBL1XR1 primary antibody (1:400, ab117761, Abcam, USA) at $4^{\circ} \mathrm{C}$ overnight. The immunostaining was achieved after incubation with biotinylated goat anti-mouse secondary antibody using avidin-biotin complex (ABC) method. Slides were finally mounted with neutral balsam after hematoxylin counter-staining and dehydrated. 5\% fetal bovine serum (BSA) was used as a negative control.

IHC results were scored based on staining intensity $(0=$ negative, 1 = weak, 2 = moderate, $3=$ strong) and the percentage of stained tumor cells $(0 \leq 20 \%, 1=21-50 \%, 2=51-80 \%, 3=81-100 \%)$ by two pathologists, independently. The final IHC score was obtained by multiplying intensity score with percentage score, which ranges from 0 to 9 . Slides with IHC score less than 4 were grouped as low expression, while those with IHC score $\geq 4$ were grouped as high expression.

Protein extraction and western blot analysis

Clinical tissues or cultured cells were lysed with RIPA buffer (Beyotime Biotechnology, China). Equal amounts of proteins were separated by $10 \%$ polyacrylamide gel electrophoresis (SDS-PAGE) and transferred onto Immobilon-P Transfer membrane (Merck Millipore, China). Immunoblots were prepared using standard protocols including BSA blocking, primary antibody incubation (VEGF-C, sc-374628; $\beta$-actin, sc-58673; both from Santa Cruz Biotechnology, USA), secondary horseradish peroxidase-conjugated antibody incubation (anti-goat IgG for VEGF-C, anti-mouse IgG for TBL1XR1 and $\beta$-actin) and eventually developed with chemiluminescence reagents (Santa Cruz Biotechnology, USA).

\section{$R N A$ extraction and real-time quantitative PCR (RT-qPCR)}

Total RNA was extracted with TRIzol reagent (Invitrogen, California, USA) according to the manufacturer's instruction. The total RNA concentration was assessed by a Nano-Drop spectrophotometer (Thermo Scientific, Pennsylvania, USA), and reverse transcription was performed with $1 \mu \mathrm{g}$ of total RNA using reverse transcriptase (Promega, USA). The complemental cDNA was then subjected to RT-qPCR to analyze mRNA levels of TBL1XR1 and VEGF-C. The PCR cycle included preheating at $95^{\circ} \mathrm{C}$ for $10 \mathrm{~min}$ and then amplified at $95^{\circ} \mathrm{C}(30 \mathrm{sec})$ and $60^{\circ} \mathrm{C}(1 \mathrm{~min})$ for 45 cycles. The threshold cycle $(\mathrm{Ct})$ value of each sample was calculated and the relative mRNA levels were normalized to the glyceraldehyde 3-phosphate dehydrogenase (GAPDH) level. The data were evaluated with $2^{-4 \mathrm{Ct}}$ method (Bustin et al. 2009). The primers are as followed:

TBL1XR1: 5'-GAG GTG TTT ATT TGT GCT TGG-3' and 5'-TGC ACT TAA TAT GAA GTT GCC-3'; VEGF-C: 5'-GTG ACC AAC ATG GAG TCG TG-3' and 5'-CCA GAG ATT CCA TGC CAC TT; GAPDH: 5'-GAA GGT GAA GGT CGG AGT C-3' and 5'-CCC GAA TCA CAT TCT CCA AGA A-3'.

\section{Cell culture and transfection}

The ovarian benign cell line Hs893 was purchased from American Type Culture Collection (ATCC, USA). The human epithelial ovarian cancer cell lines (OVCAR3 and SKOV3) were obtained from the Cell Bank of the Chinese Academy of Sciences, Shanghai, China. All cells were cultured in RPMI 1640 medium supplemented with $10 \%(\mathrm{v} / \mathrm{v})$ fetal bovine serum (FBS), $100 \mathrm{U} / \mathrm{mL}$ penicillin and $100 \mu \mathrm{g} / \mathrm{mL}$ streptomycin.

The Myc-tagged human TBL1XR1 cDNA was obtained from Origene (NM_024665) and cloned into pcDNA3.0 vector for trans- 
fection. TBL1XR1 knockdown was performed by oligo-siRNA transient transfection with the siRNA sequences below:

TBL1XR1 siRNA\#1: 5'-TCT CTA GAT TGG AAT AGT GAAdTdT-3' (Guo et al. 2016)

TBL1XR1 siRNA\#2: 5'-CGT AAT GCC TGA TGT AGT

AdTdT-3' (Wang et al. 2014)

Negative control siRNA (ncRNA): 5'-TTC TCC GAA CGT

GTC ACG TdTdT-3'

\section{Cell viability assay}

Presto-Blue cell viability reagent (Invitrogen, Life Technologies, USA) was used to test the cell viability of OVCAR3 and SKOV3 cells. Briefly, cells were seeded at $1 \times 10^{4}$ cells/well in 96-well plates after transfection, and Presto-Blue reagent was added at the designated time points. The fluorescence was obtained at a wavelength of $560 \mathrm{~nm}$ with a microplate reader. Each experiment was performed in triplicate.

Transwell invasion assay

The invasion capacity of SKOV3 and OVCAR3 cells was assessed using the Boyden chamber Matrigel invasion assay (Cell Biolabs, USA) as described before (Ma and Yu 2016). Briefly, $1 \times 10^{5}$ transfected cells were planted into the upper chamber $24 \mathrm{~h}$ after transfection. DMEM containing 1\% FBS was added into the upper chamber, while DMEM with $10 \%$ FBS was added into the lower chamber. After another 48-h incubation, non-invaded cells were removed gently with a cotton swab, and migrated cells to the lower surface of membrane were then fixed with methanol and stained with crystal violet. Cell counting was performed in five randomly selected fields for each well. Each experiment was performed in triplicate. date of surgery to the date of death or the last date of follow-up (September 2015). The 5-year OS was calculated as the proportion of patients who survived for more than 5 years after surgery. Statistical analysis was performed with SPSS 22.0 software (SPSS, Chicago, IL, USA). The associations between TBL1XR1 expression and other clinicopathological parameters were assessed with chi-square test. Univariate survival analysis was performed using the Kaplan-Meier method and log-rank test. The Cox proportional hazard regression analysis was employed for multivariate survival analysis. A P value of less than 0.05 was considered as statistically significant (twosided).

\section{Results}

\section{Patient characteristics}

Our study included 116 patients aged from 46 to 75 years (median 54.5 years), and the median follow up time was 59 months. The 5 -year OS of the entire cohort was $70.3 \%$. The histopathologic grading of the 116 serous EOC patients revealed that 35 patients $(30.2 \%)$ were with poor differentiation, and the other 81 patients $(69.8 \%)$ were with well or moderate differentiation. Patients' clinical stages were determined according to the FIGO staging system. In our cohort, 12 cases $(10.3 \%)$ had stage I, 21 cases $(18.1 \%)$ had stage II, 62 cases (53.4\%) had stage III, and 21 cases (18.1\%) had stage IV. Among the 116 serous EOC patients, 62 patients $(53.4 \%)$ were diagnosed as positive lymph node metastasis, and the other 54 patients $(46.6 \%)$ had negative lymph node metastasis. Detailed characteristics of patients are summarized in Table 1.

\section{Statistical analysis}

The overall survival (OS) was defined as the period from the

Table 1. Expression of TBL1XR1 in serous EOC tissues.

\begin{tabular}{|c|c|c|c|c|}
\hline \multirow[t]{2}{*}{ Variables } & \multirow{2}{*}{$\begin{array}{c}\text { Cases } \\
(\mathrm{n}=116)\end{array}$} & \multicolumn{2}{|c|}{ TBL1XR1 expression } & \multirow[t]{2}{*}{$P$ value } \\
\hline & & Low $(n=62)$ & High $(\mathrm{n}=54)$ & \\
\hline Age (years) & & & & 0.519 \\
\hline$\leq 60$ & 87 & 48 & 39 & \\
\hline$>60$ & 29 & 14 & 15 & \\
\hline FIGO stage & & & & $0.009^{*}$ \\
\hline $\mathrm{I} \sim \mathrm{II}$ & 33 & 24 & 9 & \\
\hline $\mathrm{III} \sim \mathrm{IV}$ & 83 & 38 & 45 & \\
\hline Pathological differentiation & & & & $0.021 *$ \\
\hline Well/Moderate & 81 & 49 & 32 & \\
\hline Poor & 35 & 13 & 22 & \\
\hline Lymph node metastasis & & & & $0.002 *$ \\
\hline Negative & 54 & 37 & 17 & \\
\hline Positive & 62 & 25 & 37 & \\
\hline Preoperative serum CA-125 & & & & 0.265 \\
\hline$\leq 822 \mathrm{U} / \mathrm{mL}$ & 58 & 27 & 31 & \\
\hline$>822 \mathrm{U} / \mathrm{mL}$ & 58 & 33 & 25 & \\
\hline
\end{tabular}

EOC, epithelial ovarian cancer; FIGO, International Federation of Gynecology and Obstetrics; TBL1XR1, transducin $(\beta)$-like 1 X-linked receptor 1. 
TBL1XR1 is highly expressed in EOC tissues and correlated with lymph node metastasis

IHC results showed that TBL1XR1 was mainly localized in the nucleus of the EOC cells (Fig. 1A, B). Among the 116 serous patients, 54 cases $(46.6 \%)$ were identified with high TBL1XR1 expression, whereas the other 62 patients $(53.4 \%)$ were with low TBL1XR1 expression. The correlations between the expression of TBL1XR1 and other clinicopathologic characters are shown in Table 1. In addition, western blot analysis of the fresh-frozen tissues demonstrated that the protein levels of TBL1XR1 were higher in tumor tissues than in adjacent non-tumorous tissues (Fig. 1C, D). Consistently, RT-qPCR results showed that the mRNA levels of TBL1XR1 were also higher in tumor tissues (Fig. 1E).

For the 83 cases with FIGO stage III-IV, 45 patients $(45 / 83,54.2 \%)$ were classified as TBL1XR1 high expression; while only 9 patients $(9 / 33,27.3 \%)$ with FIGO stage I-II were identified with TBL1XR1 high expression (Fig. 2A). In addition, TBL1XR1 expression level was significantly correlated with the status of lymph node metastasis
$(\mathrm{P}=0.002$, Table 1, Fig. 2B). The correlations revealed that TBL1XR1 may play an essential role in the process of lymph node metastasis. Patients with poor pathological differentiation tumors also showed higher TBL1XR1 levels ( $P$ $=0.021$, Table 1$)$. No statistically significant correlation was observed between TBL1XR1 expression and age $(\mathrm{P}=$ $0.519)$ or pre-operative CA125 level $(\mathrm{P}=0.265)$.

TBL1XR1 is an independent prognostic factor for the OS of serous EOC patients

The 5-year OS for the whole cohort was 70.3\%, and the median survival time was 71 months. Kaplan-Meier univariate survival analysis revealed that age $(\mathrm{P}=0.042)$, FIGO stage ( $\mathrm{P}=0.026)$, lymph node metastasis $(\mathrm{P}=0.009)$, and TBL1XR1 expression $(\mathrm{P}=0.011)$ were all significantly associated with the OS of serous EOC patients. Patients with high TBL1XR1 expression showed unfavorable prognosis compared to those with low TBL1XR1 expression (OS $75.7 \pm 5.3$ vs. $105.9 \pm 7.3$ months; $\mathrm{P}=0.011$; Fig. $2 \mathrm{C}$, Table 2).

Furthermore, Cox-regression multivariate analysis
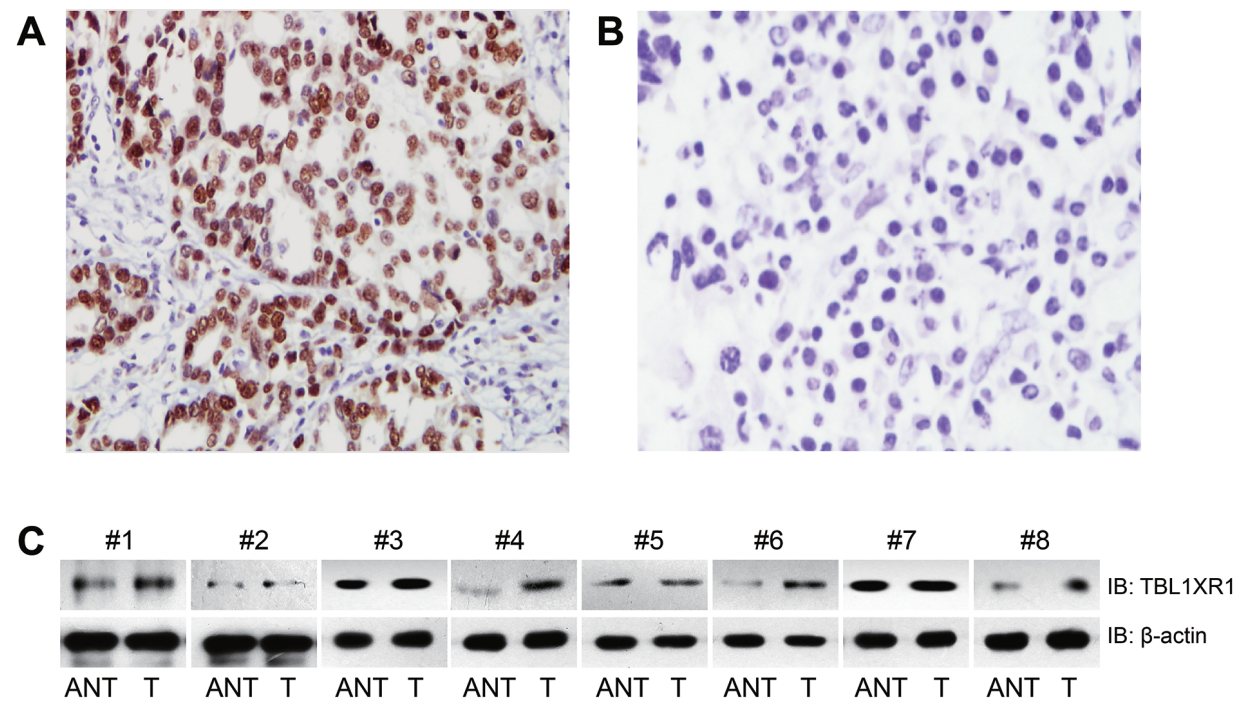

D

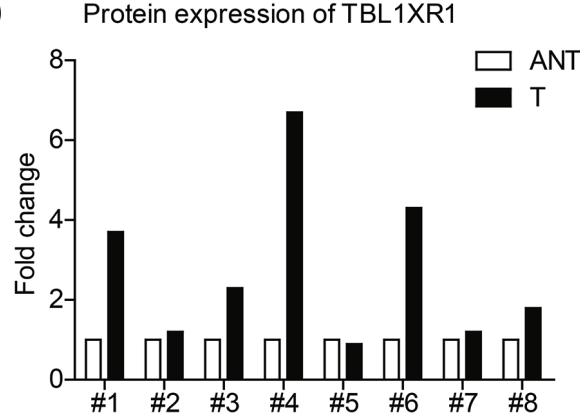

E mRNA level of TBL1XR1

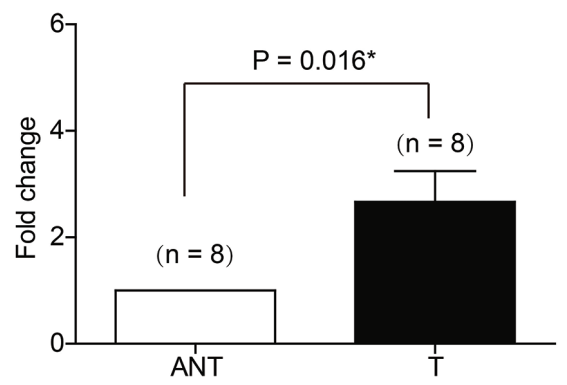

Fig. 1. Expression of TBL1XR1 in serous EOC tissues.

(A) Representative high expression of TBL1XR1 in EOC tissues, with the IHC score 9. (B) Representative low expression of TBL1XR1 in EOC tissues, with the IHC score 0. (C) Western blot analysis showed the protein levels of TBL1XR1 in 8 pairs of EOC tissues (T) and adjacent non-tumorous tissues (ANT). (D) Quantification of the protein levels of TBL1XR1 with Graphpad prism 5.0 software. (E) RT-qPCR results demonstrated that the mRNA level of TBL1XR1 was higher in tumor tissues than that in adjacent non-tumorous tissues $(n=8)$. Magnification: $200 X$. 
A TBL1XR1 expression for different FIGO stages

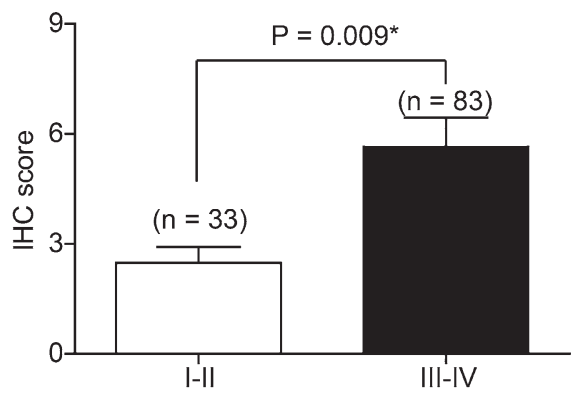

C

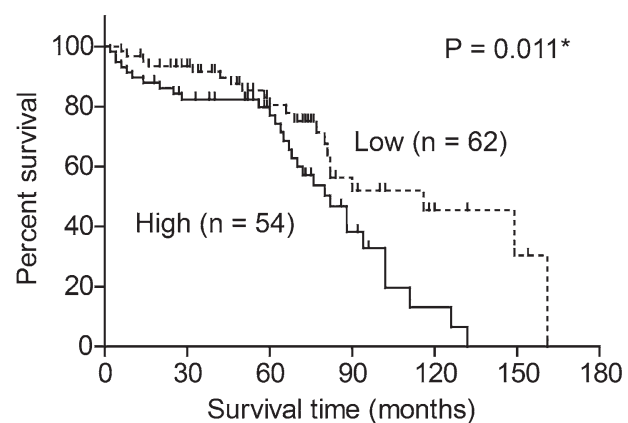

B TBL1XR1 expression for different LN status

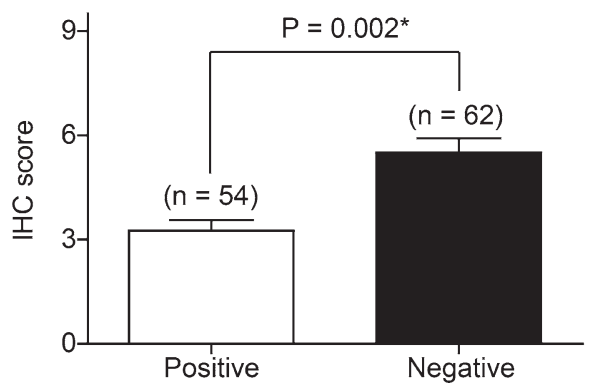

D Correlation between TBL1XR1 and VEGF-C

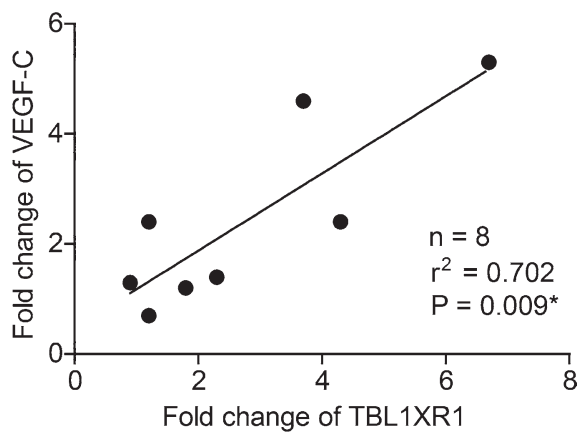

Fig. 2. Correlations between TBL1XR1 and clinical characteristics of serous EOC patients.

(A) EOC patients with advanced FIGO stages showed higher immunoreactivity of TBL1XR1 (P=0.009). (B) Expression levels of TBL1XR1 were higher in patients with positive lymph node metastasis than in those with negative lymph node metastasis $(\mathrm{P}=0.002)$. (C) Kaplan-Meier survival curve showed that higher expression of TBL1XR1 was correlated with poorer overall survival of EOC patients. (D) The VEGF-C mRNA levels were positively correlated with TBL1XR1 mRNA expression in tumor tissues (The data was generated by RT-qPCR from 8 paired EOC tissues and adjacent non-tumorous tissues. $\mathrm{X}$ axis showed the fold changes of TBL1XR1 while $\mathrm{Y}$ axis showed the fold changes of VEGF-C in corresponding tissues).

indicated that TBL1XR1 was as an independent prognostic factor (hazard ratio $[\mathrm{HR}]=2.006$, confidence interval $[\mathrm{CI}]$ $=1.294-5.267, \mathrm{P}=0.038$ ). In addition, advanced FIGO stage $(\mathrm{HR}=2.766, \mathrm{CI}=1.481-4.926, \mathrm{P}=0.001)$ and positive lymph node metastasis $(\mathrm{HR}=2.125, \mathrm{CI}=1.184-3.225$, $\mathrm{P}=0.026$ ) were both negatively correlated with OS (Table $3)$.

\section{TBL1XR1 positively regulates the expression of VEGF-C}

The RT-qPCR results from fresh-frozen tissues showed that the mRNA expression level of VEGF-C was positively correlated with that of TBL1XR1 $\left(\mathrm{r}^{2}=0.702, \mathrm{P}=0.009\right.$, Fig. 2D). To better explore the functional mechanisms of TBL1XR1 in EOC cells, we analyzed the protein expression of TBL1XR1 in both normal ovarian epithelial cells (Hs832) and EOC cell lines (SKOV3 and OVCAR3). The highest expression level of TBL1XR1 was found in OVCAR3 cells among three cell lines, and there was no significant difference between $\mathrm{Hs} 832$ and SKOV3 cells (Fig. 3A, B). Therefore, we next overexpressed TBL1XR1 in SKOV3 cells, and silenced TBL1XR1 expression in OVCAR3 cells. The transfection efficiencies were confirmed by western blot analysis. Moreover, the expression of VEGF-C showed similar patterns correspondingly with the protein level of TBL1XR1 (Fig. 3C-F).

High expression of TBL1XR1 promotes the viability and invasion of EOC cells

Since VEGF-C plays a critical role in the lymph node metastasis of tumors, we therefore performed cellular assays to investigate whether TBL1XR1 can influence the proliferation and invasion capacities of EOC cells. The viability of cultured cells was detected by Presto-Blue cell viability reagent. We chose SKOV3 cells to compare the differences in cell proliferation among transfections with TBL1XR1, pcDNA3.0-vector and blank control. TBL1XR1 overexpression significantly enhanced the proliferation of EOC cells (Fig. 4A). In contrast, TBL1XR1-siRNA showed inhibitory effect on OVCAR3 proliferation (Fig. 4B).

We also performed Transwell assay to see whether TBL1XR1 can modulate the cell invasion capacity. The invasion results showed that TBL1XR1 overexpression can remarkably increase the cell invasion by 1.7 folds (Fig. 4C), whereas TBL1XR1 silencing reduced cell invasion by $40 \%$ $60 \%$ (Fig. 4D). These findings demonstrated that TBL1XR1 can positively regulate the invasion of ovarian cancer cell viability. 
Table 2. Kaplan-Meier survival analysis (log-rank test) of serous EOC patients.

\begin{tabular}{|c|c|c|c|c|}
\hline Variables & $\begin{array}{c}\text { Cases } \\
(\mathrm{n}=116)\end{array}$ & $\begin{array}{c}\text { 5-year OS } \\
\text { rate }(\%)\end{array}$ & $\begin{array}{l}\text { OS (months) } \\
\text { Mean } \pm \text { S.D. }\end{array}$ & $P$ value \\
\hline Age (years) & & & & $0.042 *$ \\
\hline$\leq 60$ & 87 & $83.1 \%$ & $99.4 \pm 6.5$ & \\
\hline$>60$ & 29 & $71.2 \%$ & $71.5 \pm 5.7$ & \\
\hline FIGO stage & & & & $0.026^{*}$ \\
\hline $\mathrm{I} \sim \mathrm{II}$ & 33 & $86.4 \%$ & $99.4 \pm 5.6$ & \\
\hline III $\sim$ IV & 83 & $62.7 \%$ & $85.7 \pm 11.2$ & \\
\hline Pathological differentiation & & & & 0.114 \\
\hline Well/Moderate & 71 & $91.2 \%$ & $101.7 \pm 8.0$ & \\
\hline Poor & 35 & $73.0 \%$ & $88.4 \pm 6.6$ & \\
\hline Lymph node metastasis & & & & $0.009^{*}$ \\
\hline Negative & 54 & $90.6 \%$ & $108.2 \pm 6.3$ & \\
\hline Positive & 62 & $69.8 \%$ & $73.2 \pm 5.8$ & \\
\hline Preoperative serum CA-125 & & & & 0.417 \\
\hline$\leq 822 \mathrm{U} / \mathrm{mL}$ & 58 & $81.2 \%$ & $97.4 \pm 8.1$ & \\
\hline$>822 \mathrm{U} / \mathrm{mL}$ & 58 & $77.8 \%$ & $89.5 \pm 6.8$ & \\
\hline TBL1XR1 expression & & & & $0.011 *$ \\
\hline Low & 62 & $80.5 \%$ & $105.9 \pm 7.3$ & \\
\hline High & 54 & $77.0 \%$ & $75.7 \pm 5.3$ & \\
\hline
\end{tabular}

EOC, epithelial ovarian cancer; FIGO, International Federation of Gynecology and Obstetrics; TBL1XR1, transducin $(\beta)$-like 1 X-linked receptor 1.

Table 3. Cox multivariate an $\mathrm{g}$ alysis for serous EOC patients.

\begin{tabular}{lccc}
\hline Variables & Hazard ratio & $95 \%$ Confidence Interval & P value \\
\hline Age & 1.460 & $0.954-2.791$ & 0.175 \\
FIGO stage & 2.766 & $1.481-4.926$ & $0.001^{*}$ \\
Lymph node metastasis & 2.125 & $1.184-3.225$ & $0.026^{*}$ \\
TBL1XR1 expression & 2.006 & $1.294-5.267$ & $0.038^{*}$ \\
\hline
\end{tabular}

EOC, epithelial ovarian cancer; FIGO, International Federation of Gynecology and Obstetrics; TBL1XR1, transducin $(\beta)$-like 1 X-linked receptor 1.

\section{Discussion}

EOC accounts for $80 \%-90 \%$ of ovarian cancers and is one of the leading causes of death in patients with gynecologic malignancies (Siegel et al. 2014). The absence of specific symptoms and lack of reliable early diagnostic methods result in the diagnosis of $70 \%$ of patients at an advanced stage (Lan et al. 2009). EOC, as well as other tumors, is thought to arise from the accumulation of genetic alterations (Aunoble et al. 2000). Therefore, understanding the molecular mechanisms and searching for novel biomarkers involved in the progression of EOC are invaluable for the prediction of clinical outcomes, providing novel therapeutic targets, and improving patients' survival.

The role of TBL1XR1 on mediating estrogen receptors (ER) signaling in ovarian cancer cell lines has been reported recently, which identified it as an ER corepressor in regulating oncogenicity of ovarian cancer cells (Wu et al. 2016).
Here, in the present study, we found that the expression levels of TBL1XR1 in ovarian cancer tissues were higher than that in normal ovarian tissues, and high levels of TBL1XR1 expression were associated with aggressive clinical conditions in ovarian cancer. Moreover, we demonstrated that high TBL1XR1 levels indicated poor prognosis of serous EOC patients. To our knowledge, this is the first report about the role of TBL1XR1 in predicting prognosis of ovarian cancer. Finally, our cellular experiments demonstrated that knock-down of TBL1XR1 can inhibit ovarian cancer cell growth and invasion.

EOC patients with positive lymph node metastasis show unfavorable overall survival, and the status of lymph node is the most important factor for prognosis predicting (Siegel et al. 2015). However, it is sometimes not easy to determine lymph node status and the false negative rate is not satisfied. Therefore, biomarkers that can help to predict prevalence of lymph node metastasis may be helpful for the 


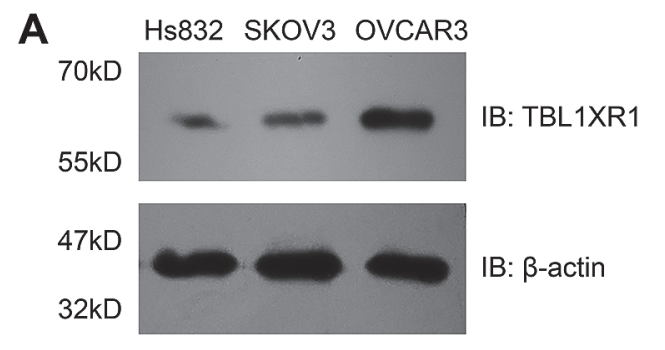

B TBL1XR1 expression in different cell lines
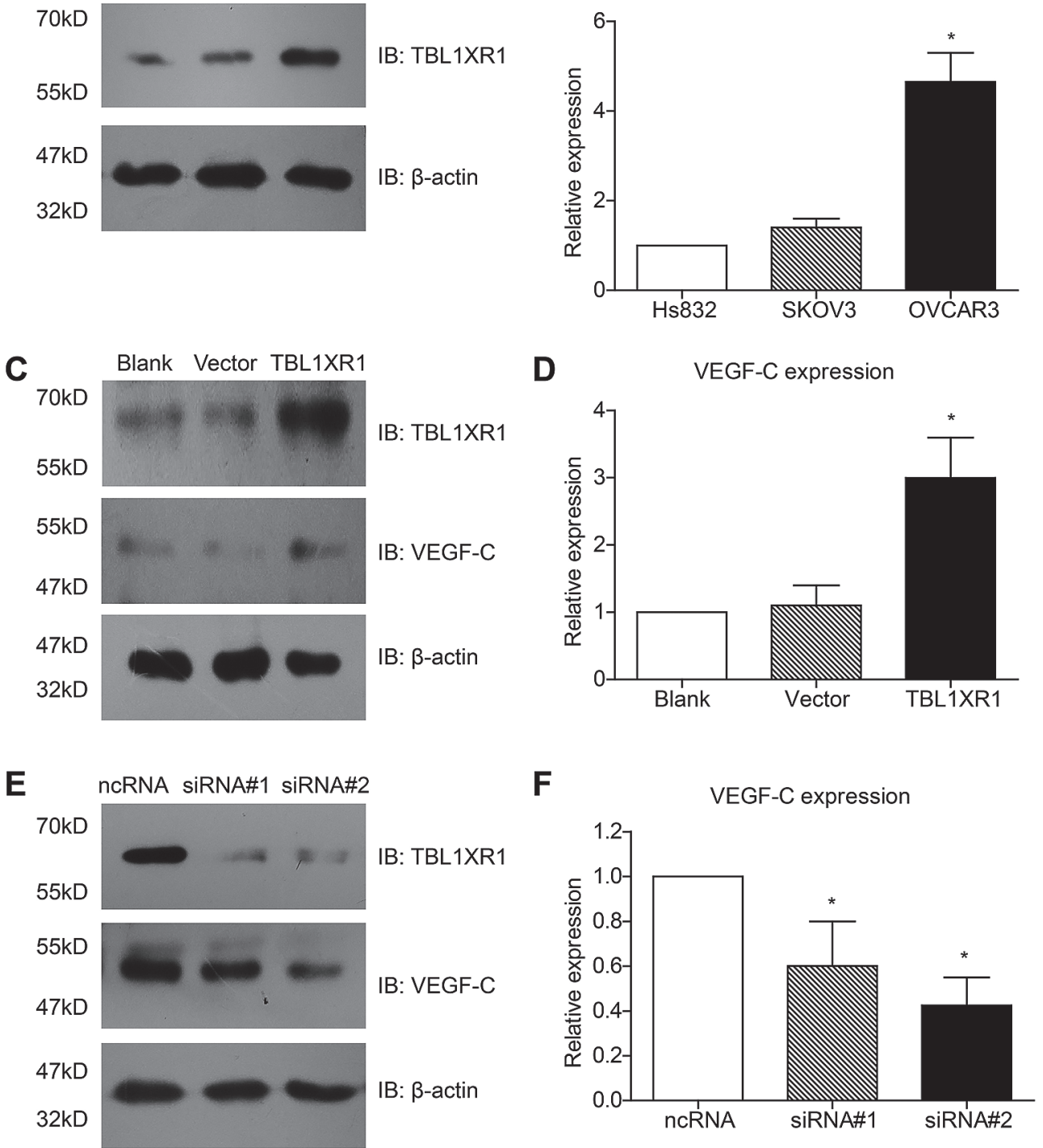

Fig. 3. TBL1XR1 modulates the expression of VEGF-C in EOC cells.

TBL1XR1 showed highest protein expression in OVCAR3 cells among Hs832, SKOV3, and OVCAR3 cell lines, whereas no significant difference was observed between Hs832 and SKOV3 cells (A, B). TBL1XR1 overexpression increased the protein level of VEGF-C (C, D). In contrast, TBL1XR1 silencing by siRNAs down-regulated the protein expression of VEGF-C (E, F). Each experiment was performed in triplicate.

clinical evaluation. VEGF-C, a subtype of VEGF family, has been reported to participate in the lymphatic metastasis of various solid tumors including ovarian cancers (Bednarek et al. 2008; Wu et al. 2012; Regan et al. 2016; Wang et al. 2016). Interestingly, our study revealed that the mRNA expression of VEGF-C was positively correlated with TBL1XR1 levels in clinical tumor tissues. Moreover, overexpression and silencing experiments demonstrated that the VEGF-C expression was modulated by TBL1XR1 in EOC cell lines. In other words, VEGF-C is likely to be a downstream effecter of TBL1XR1 in ovarian cancer. There is high possibility that TBL1XR1 may promote tumor progression and lymphatic metastasis through VEGF-C pathways, although more detailed biomolecular studies are needed to verify it.
In conclusion, the overexpression of TBL1XR1 is an independent predictive factor for the poor prognosis of serous EOC patients. Our findings also indicate that the TBL1XR1-VEGF-C axis is important in the EOC progression. We thus suggest the potential of TBL1XR1 in the development of novel chemotherapy.

\section{Conflict of Interest}

The authors declare no conflict of interest. 
A

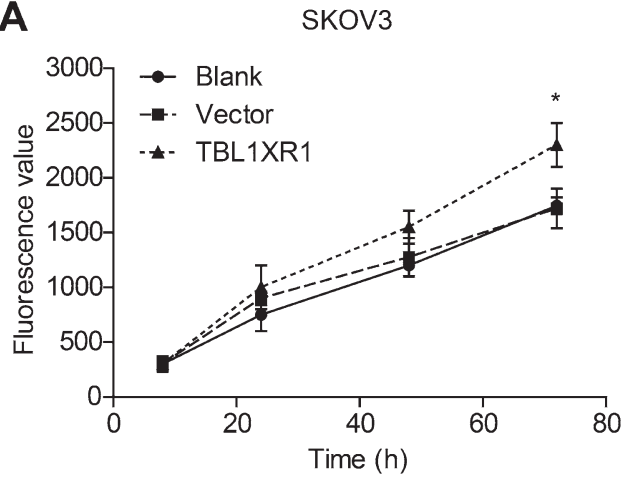

C

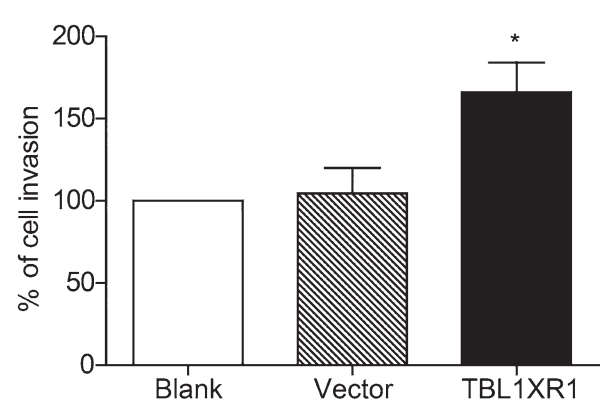

B

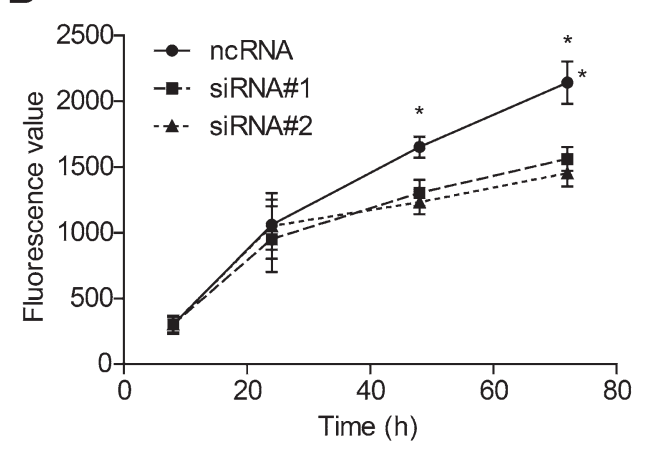

D

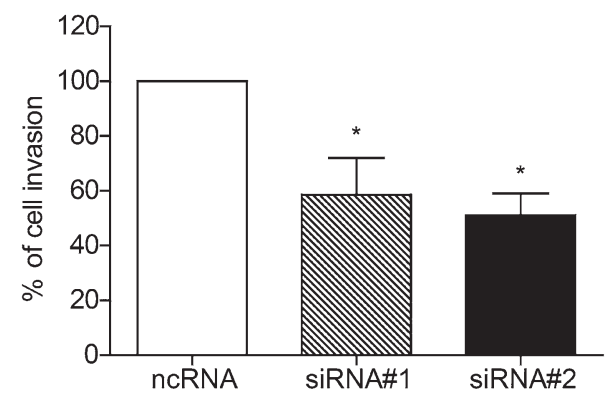

Fig. 4. Role of TBL1XR1 in regulating proliferation and invasion of EOC cells.

TBL1XR1 overexpression enhanced the proliferation ability of SKOV3 cells (A), and TBL1XR1 knock-down impaired the viability of OVCAR3 cells (B). Similarly, the invasion capacity of EOC cells was also positively correlated with the TBL1XR1 expression levels (C, D). Each experiment was performed in triplicate.

\section{References}

Aunoble, B., Sanches, R., Didier, E. \& Bignon, Y.J. (2000) Major oncogenes and tumor suppressor genes involved in epithelial ovarian cancer (review). Int. J. Oncol., 16, 567-576.

Bednarek, W., Wertel, I. \& Kotarski, J. (2008) Lymphangiogenesis in cancerous tumours. Ginekol. Pol., 79, 625-629.

Bustin, S.A., Benes, V., Garson, J.A., Hellemans, J., Huggett, J., Kubista, M., Mueller, R., Nolan, T., Pfaffl, M.W., Shipley, G.L., Vandesompele, J. \& Wittwer, C.T. (2009) The MIQE guidelines: minimum information for publication of quantitative real-time PCR experiments. Clin. Chem., 55, 611-622.

Chen, S.P., Yang, Q., Wang, C.J., Zhang, L.J., Fang, Y., Lei, F.Y., Wu, S., Song, L.B., Guo, X. \& Guo, L. (2014) Transducin $\beta$-like $1 \mathrm{X}$-linked receptor 1 suppresses cisplatin sensitivity in nasopharyngeal carcinoma via activation of NF- $\kappa \mathrm{B}$ pathway. Mol. Cancer, 13, 195.

Choi, H.K., Choi, K.C., Yoo, J.Y., Song, M., Ko, S.J., Kim, C.H., Ahn, J.H., Chun, K.H., Yook, J.I. \& Yoon, H.G. (2011) Reversible SUMOylation of TBL1-TBLR1 regulates $\beta$-catenin-mediated Wnt signaling. Mol. Cell, 43, 203-216.

Colombo, P.E., Fabbro, M., Theillet, C., Bibeau, F., Rouanet, P. \& Ray-Coquard, I. (2014) Sensitivity and resistance to treatment in the primary management of epithelial ovarian cancer. Crit. Rev. Oncol. Hematol., 89, 207-216.

Daniels, G., Li, Y., Gellert, L.L., Zhou, A., Melamed, J., Wu, X., Zhang, X., Zhang, D., Meruelo, D., Logan, S.K., Basch, R. \& Lee, P. (2014) TBLR1 as an androgen receptor (AR) coactivator selectively activates AR target genes to inhibit prostate cancer growth. Endocr. Relat. Cancer, 21, 127-142.

Daniels, G., Zhang, X., Zhong, X., Santiago, L., Wang, L.H., Wu, X., Zhang, J.Y., Liang, F., Li, X., Neubert, T.A., Steinke, L.,
Shen, Y., Basch, R., Schneider, R., Levy, D.E., et al. (2016) Cytoplasmic, full length and novel cleaved variant, TBLR1 reduces apoptosis in prostate cancer under androgen deprivation. Oncotarget, 7, 39556-39571.

Goff, B.A., Mandel, L., Muntz, H.G. \& Melancon, C.H. (2000) Ovarian carcinoma diagnosis. Cancer, 89, 2068-2075.

Guo, Y., Wang, J., Zhang, L., Shen, S., Guo, R., Yang, Y., Chen, W., Wang, Y., Chen, G. \& Shuai, X. (2016) Theranostical nanosystem-mediated identification of an oncogene and highly effective therapy in hepatocellular carcinoma. Hepatology, 63, 1240-1255.

Hoberg, J.E., Yeung, F. \& Mayo, M.W. (2004) SMRT derepression by the IkappaB kinase alpha: a prerequisite to NF-kappaB transcription and survival. Mol. Cell, 16, 245-255.

Hunn, J. \& Rodriguez, G.C. (2012) Ovarian cancer: etiology, risk factors, and epidemiology. Clin. Obstet. Gynecol., 55, 3-23.

Kuang, X., Zhu, J., Peng, Z., Wang, J. \& Chen, Z. (2016) Transducin (Beta)-Like 1 X-Linked Receptor 1 Correlates with Clinical Prognosis and Epithelial-Mesenchymal Transition in Hepatocellular Carcinoma. Dig. Dis. Sci., 61, 489-500.

Lan, C., Li, Y. \& Liu, J. (2009) Intraperitoneal access via direct puncture is an alternative way to deliver intraperitoneal chemotherapy in ovarian, fallopian tube and primary peritoneal cancer. Gynecol. Oncol., 114, 42-47.

Li, J. \& Wang, C.Y. (2008) TBL1-TBLR1 and beta-catenin recruit each other to Wnt target-gene promoter for transcription activation and oncogenesis. Nat. Cell Biol., 10, 160-169.

Li, X., Liang, W., Liu, J., Lin, C., Wu, S., Song, L. \& Yuan, Z. (2014) Transducin (beta)-like $1 \mathrm{X}$-linked receptor 1 promotes proliferation and tumorigenicity in human breast cancer via activation of beta-catenin signaling. Breast Cancer Res., 16, 465.

Liu, F., He, Y. \& Cao, Q. (2016a) TBL1XR1 Is Highly Expressed 
in Gastric Cancer and Predicts Poor Prognosis. Dis. Markers, 2016, 2436518.

Liu, H., Xu, Y., Zhang, Q., Yang, H., Shi, W., Liu, Z., Li, K., Gong, Z., Ning, S., Li, S. \& Chen, Y. (2017) Prognostic significance of TBL1XR1 in predicting liver metastasis for early stage colorectal cancer. Surg. Oncol., 26, 13-20.

Liu, H., Zhang, Q., Li, K., Gong, Z., Liu, Z., Xu, Y., Swaney, M.H., Xiao, K. \& Chen, Y. (2016b) Prognostic significance of USP33 in advanced colorectal cancer patients: new insights into beta-arrestin-dependent ERK signaling. Oncotarget, 7, 81223-81240.

Ma, M. \& Yu, N. (2016) Ubiquitin-specific protease 7 expression is a prognostic factor in epithelial ovarian cancer and correlates with lymph node metastasis. Onco Targets Ther., 9, 1559-1569.

Perissi, V., Scafoglio, C., Zhang, J., Ohgi, K.A., Rose, D.W., Glass, C.K. \& Rosenfeld, M.G. (2008) TBL1 and TBLR1 phosphorylation on regulated gene promoters overcomes dual $\mathrm{CtBP}$ and NCoR/SMRT transcriptional repression checkpoints. Mol. Cell, 29, 755-766.

Regan, E., Sibley, R.C., Cenik, B.K., Silva, A., Girard, L., Minna, J.D. \& Dellinger, M.T. (2016) Identification of Gene Expression Differences between Lymphangiogenic and NonLymphangiogenic Non-Small Cell Lung Cancer Cell Lines. PLoS One, 11, e0150963.

Siegel, R., Ma, J., Zou, Z. \& Jemal, A. (2014) Cancer statistics, 2014. CA Cancer J. Clin., 64, 9-29.

Siegel, R., Naishadham, D. \& Jemal, A. (2012) Cancer statistics for Hispanics/Latinos, 2012. CA Cancer J. Clin., 62, 283-298.

Siegel, R.L., Miller, K.D. \& Jemal, A. (2015) Cancer statistics, 2015. CA Cancer J. Clin., 65, 5-29.

Tomita, A., Buchholz, D.R., Obata, K. \& Shi, Y.B. (2003) Fusion protein of retinoic acid receptor alpha with promyelocytic leukemia protein or promyelocytic leukemia zinc finger protein recruits N-CoR-TBLR1 corepressor complex to repress transcription in vivo. J. Biol. Chem., 278, 3078830795.

Tomita, A., Buchholz, D.R. \& Shi, Y.B. (2004) Recruitment of N-CoR/SMRT-TBLR1 corepressor complex by unliganded thyroid hormone receptor for gene repression during frog development. Mol. Cell. Biol., 24, 3337-3346.

Wang, J., Ou, J., Guo, Y., Dai, T., Li, X., Liu, J., Xia, M., Liu, L. \& He, M. (2014) TBLR1 is a novel prognostic marker and promotes epithelial-mesenchymal transition in cervical cancer. Br. J. Cancer, 111, 112-124.

Wang, L.H., Lin, C.Y., Liu, S.C., Liu, G.T., Chen, Y.L., Chen, J.J., Chan, C.H., Lin, T.Y., Chen, C.K., Xu, G.H., Chen, S.S., Tang, C.H. \& Wang, S.W. (2016) CCL5 promotes VEGF-C production and induces lymphangiogenesis by suppressing miR-507 in human chondrosarcoma cells. Oncotarget, 7, 36896-36908.

Wen, P., Kong, R., Liu, J., Zhu, L., Chen, X., Li, X., Nie, Y., Wu, K. $\&$ Wu, J.Y. (2014) USP33, a new player in lung cancer, mediates Slit-Robo signaling. Protein Cell, 5, 704-713.

Wu, Q.W., She, H.Q., Liang, J., Huang, Y.F., Yang, Q.M., Yang, Q.L. \& Zhang, Z.M. (2012) Expression and clinical significance of extracellular matrix protein 1 and vascular endothelial growth factor-C in lymphatic metastasis of human breast cancer. BMC Cancer, 12, 47.

Wu, X., Zhan, Y., Li, X., Wei, J., Santiago, L., Daniels, G., Deng, F., Zhong, X., Chiriboga, L., Basch, R., Xiong, S., Dong, Y., Zhang, X. \& Lee, P. (2016) Nuclear TBLR1 as an ER corepressor promotes cell proliferation, migration and invasion in breast and ovarian cancer. Am. J. Cancer Res., 6, 2351-2360.

Yoon, H.G., Chan, D.W., Huang, Z.Q., Li, J., Fondell, J.D., Qin, J. \& Wong, J. (2003) Purification and functional characterization of the human N-CoR complex: the roles of HDAC3, TBL1 and TBLR1. EMBO J., 22, 1336-1346.

Yuasa-Kawada, J., Kinoshita-Kawada, M., Rao, Y. \& Wu, J.Y. (2009) Deubiquitinating enzyme USP33/VDU1 is required for Slit signaling in inhibiting breast cancer cell migration. Proc. Natl. Acad. Sci. USA, 106, 14530-14535.

Zhang, J., Kalkum, M., Chait, B.T. \& Roeder, R.G. (2002) The $\mathrm{N}-\mathrm{CoR}-\mathrm{HDAC} 3$ nuclear receptor corepressor complex inhibits the JNK pathway through the integral subunit GPS2. Mol. Cell, 9, 611-623.

Zhou, Q., Wang, X., Yu, Z., Wu, X., Chen, X., Li, J., Zhu, Z., Liu, B. \& Su, L. (2016) Transducin $(\beta)$-like 1 X-linked receptor 1 promotes gastric cancer progression via the ERK1/2 pathway. Oncogene, doi: 10.1038/onc.2016.352. [Epub ahead of print]. 